\title{
Stereospecific, Pyrylium Salt-Catalyzed $O$-Glycosylations of Phenols and Alkyl Alcohols
}

Michael Martin Nielsen ${ }^{\dagger}$, Thomas Holmstrøm ${ }^{\dagger}$ and Christian Marcus Pedersen*.

\section{Author affiliations:}

University of Copenhagen, Department of Chemistry, Universitetsparken 5, 2100 Copenhagen O, Denmark

* Corresponding author, cmp@chem.ku.dk

${ }^{\dagger} \mathrm{MMN}$ and TH contributed equally.

\begin{abstract}
Despite many years of invention, the field of carbohydrate chemistry remains rather inaccessible to non-specialists, which limits the scientific impact and reach of the discoveries made in the field. Aiming to increase the availability of stereoselective glycosylation chemistry for non-specialists, we have discovered that several commercially available pyrylium salts catalyze stereospecific $O$ glycosylations of a wide range of phenols and alkyl alcohols. This catalytic reaction utilizes trichloroacetimidates, an easily accessible and synthetically proven electrophile, takes place under air and only initiates when all three reagents are mixed, which should provide better reproducibility by non-specialists. The reaction is stereospecific, resulting in $\beta$-specific glycosylations from $\alpha-$ trichloroacetimidates, whilst an $\alpha$-selective glycosylation proceeds from $\beta$-trichloroacetimidates. A mechanistic study revealed that the reaction likely proceeds via an $S_{N}$ 2-like substitution on the protonated electrophile.
\end{abstract}

\section{Introduction}

As carbohydrates occupy a central domain of biochemistry and chemical biology, the development of strategies for selective glycosylations has been pursued by organic chemists for more than a century. ${ }^{2-8}$ The introduction of glycosyl trichloroacetimidates by Michel and Schmidt ${ }^{9}$ in the early 1980 's led to a significant acceleration in the development of catalytic glycosylations and sparked the development into diverse field of research with various catalytic glycosylation methods available. ${ }^{4}$ However, despite years of significant progress, there is still a high demand for new and more 
approachable glycosylation methods as poor reproducibility by non-specialists limits the general accessibility to the field. ${ }^{1}$

One of the key challenges in glycosylation chemistry remains obtaining high degrees of stereoselectivity across substrates and three selected stereoselective methods are shown in Figure 1Error! Reference source not found.. A widespread glycosylation method involves activation of the electrophile (or "glycosyl donor"), typically resulting in a highly reactive intermediate (Figure 1, top) with varying degrees of solvent-separation. ${ }^{10,11}$ Crich and co-workers ${ }^{12-14}$ conducted pioneering work on such high-energy intermediates and achieved highly selective reactions at low temperatures in an $S_{\mathrm{N}}$ 2-like substitution reaction, ${ }^{10,11}$ allowing even challenging $\beta$-mannosylations to proceed with high stereoselectivity.

Stereoselective glycosylations have successfully been performed by internal delivery of the nucleophile (or "glycosyl acceptor"). ${ }^{15,16}$ Toshima and Takahashi have recently introduced a method for 1,2-cis-glycosylations by boronic acid catalysis from the corresponding 1,2-anhydro sugars ${ }^{17-22}$ (Figure 1, middle) with excellent stereo- and regioselectivity. This glycosylation strategy relies on the formation of a boronic ester intermediate with the nucleophile and electrophile, resulting in a stereospecific glycosylation via an $S_{\mathrm{N}}$ reaction mechanism.

Alternatively, catalytic glycosylations can be accomplished by activation of the nucleophile (Figure 1, bottom). This particular method was pioneered by Schmidt using various reagents to form a catalyst-nucleophile complex capable of activating a trichloroacetimidate electrophile, ${ }^{23-28}$ resulting in inversion of the anomeric configuration of the electrophile. In recent years, related methods relying on activated nucleophile-catalyst-electrophile intermediates have been developed by Taylor, ${ }^{29-31}$ Toshima $^{32}$ and Jacobsen, ${ }^{33-36}$ resulting in stereospecific glycosylations. As the displacement of the anomeric leaving group is dependent on complexation with the nucleophile, this particular glycosylation strategy proceeds with relatively low formation of undesired by-products as highenergy intermediates are either not formed or very short-lived as the nucleophile is always in close proximity. However, designing or discovering catalysts capable glycosylations via such nucleophilecatalyst-electrophile complexes represent a significant challenge. 


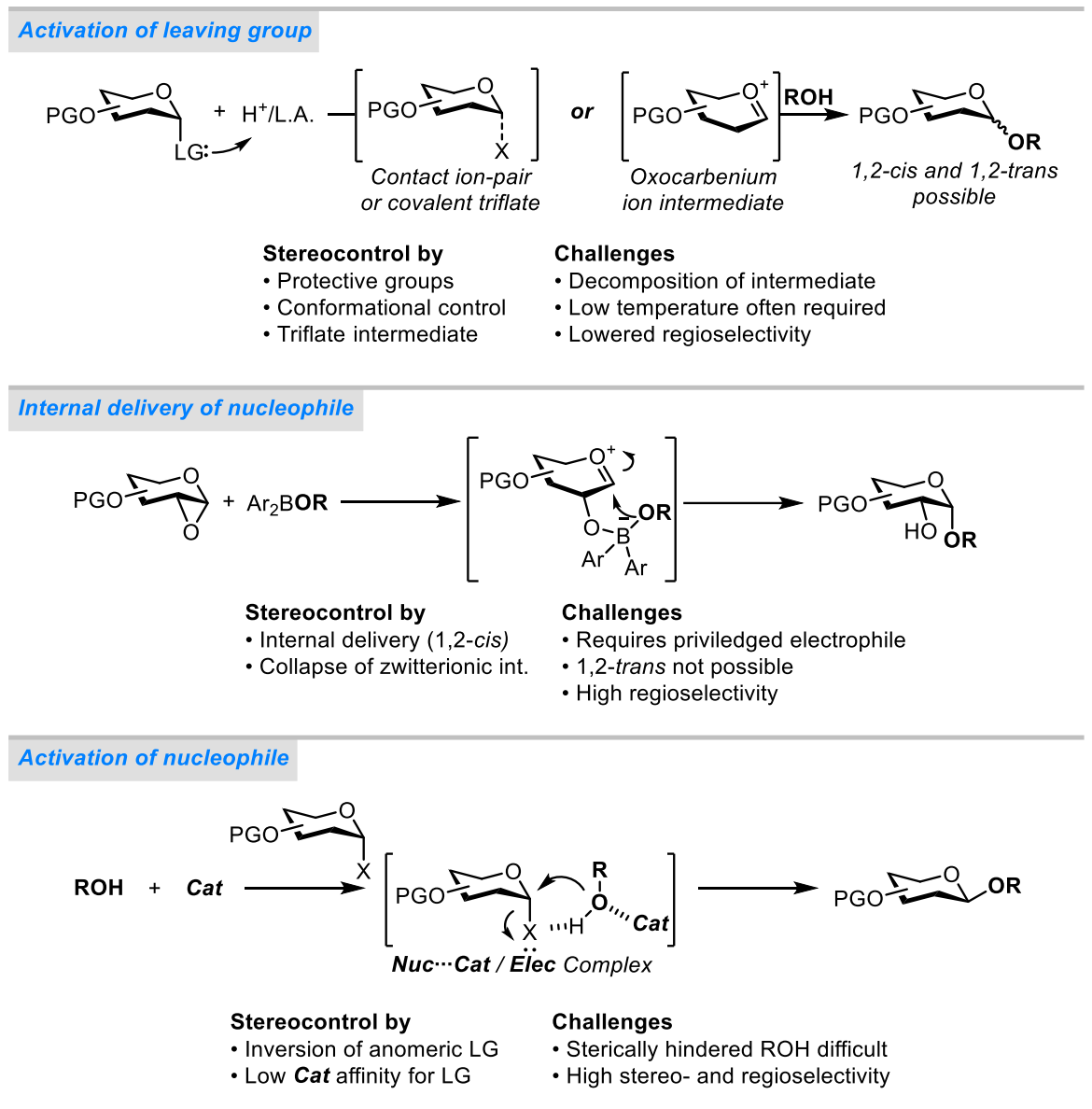

\section{[This work]}

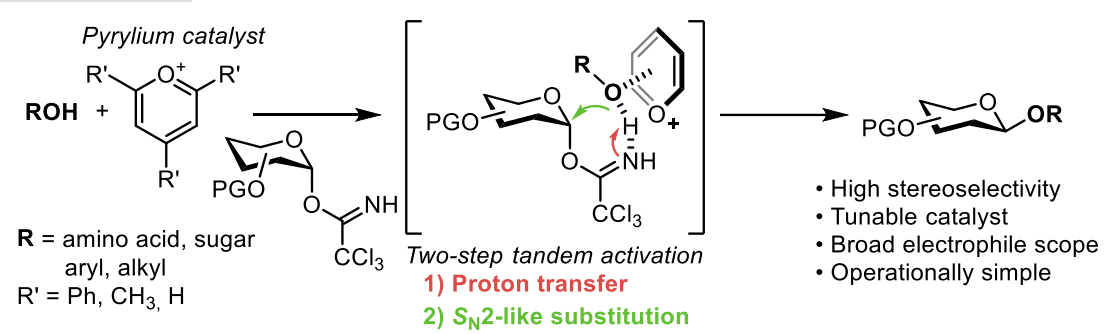

Figure 1: Graphical summary of three selected methods of stereoselective glycosylations (top, middle) and this work (bottom).

In the following, we present a new method for stereoselective $O$-glycosylations which proceeds by nucleophile activation using pyrylium catalysts (Figure 1, bottom). This discovery was made via a hypothesis-based investigation as we sought out to identify a catalytic species capable of lowering the $\mathrm{p} K_{\mathrm{a}}$ value of alcohols to the 3-5 range that we previously found ideal for stereospecific, selfpromoted $\mathrm{N}$-glycosylations. ${ }^{37,38}$ This led to the discovery that pyrylium salts are capable of providing such catalyst-nucleophile interactions, resulting in stereospecific $O$-glycosylations of trichloroacetimidate electrophiles with a wide range of phenols and alkyl alcohol nucleophiles. Many 
pyrylium salts are commercially available and have gained interest in the scientific community as organic dyes ${ }^{39,40}$ and photoredox catalysts, ${ }^{41-43}$ and were recently introduced as catalysts for Katritzky-like C-N bond activations ${ }^{44-46}$ by Cornella and co-workers. However, to the best of our knowledge, pyrylium salts have not previously been used in glycosylation chemistry.

\section{Results and discussion}

After an initial screening of reaction conditions (Table S10), it was found that three pyrylium salts were highly efficient glycosylation catalysts with both phenolic and aliphatic nucleophiles. Furthermore, the three pyrylium salts exhibited very different reaction rates during glycosylations depending on the pyrylium substitution pattern (Scheme 1).

The scope of phenolic nucleophiles was assessed using an $\alpha$-glucosyl trichloroacetimidate and 10 mol\% of the pyrylium catalyst at ambient conditions. From our survey, it was concluded that $\mathbf{H}_{3} \mathbf{P y r y}$ and Me3Pyry were significantly more reactive catalysts for the phenolic glycosylations than Ph3Pyry and furthermore resulted in higher $\beta$-selectivity. H3Pyry was found capable of catalyzing highly $\beta$ selective glycosylations of $o-, m$ - and $p$-substituted phenols (Scheme 1, products 2-5). Phenolic nucleophiles with electron-donating and -withdrawing substituents were found to give rise to similar yields and almost exclusive formation of the desired $\beta$-linked phenyl glycosides (products 6-10), which further underlines the generality of this procedure. Only a $p$-nitro-substituent (11) was found to give rise to diminished selectivity. A tyrosine side-chain was also glycosylated, yielding only the desired $\beta$-D-Glc- $O$-Tyr glycoside (12) that has been identified as a carbohydrate-protein linkage in surface-layer glycans. ${ }^{47}$ Furthermore, it was possible to prepare precursors for both D-(-)-salicin (2) and arbutin (13), two natural products containing $\beta$-glucosidic linkages. Finally, we found that several nucleophilic sites on the nucleophile is compatible with the catalytic conditions, allowing a notable chemoselective glycosylation of a phenolic nucleophile (14) in the presence of a benzylic alcohol with excellent stereoselectivity (confirmed by HMBC C-H couplings). 

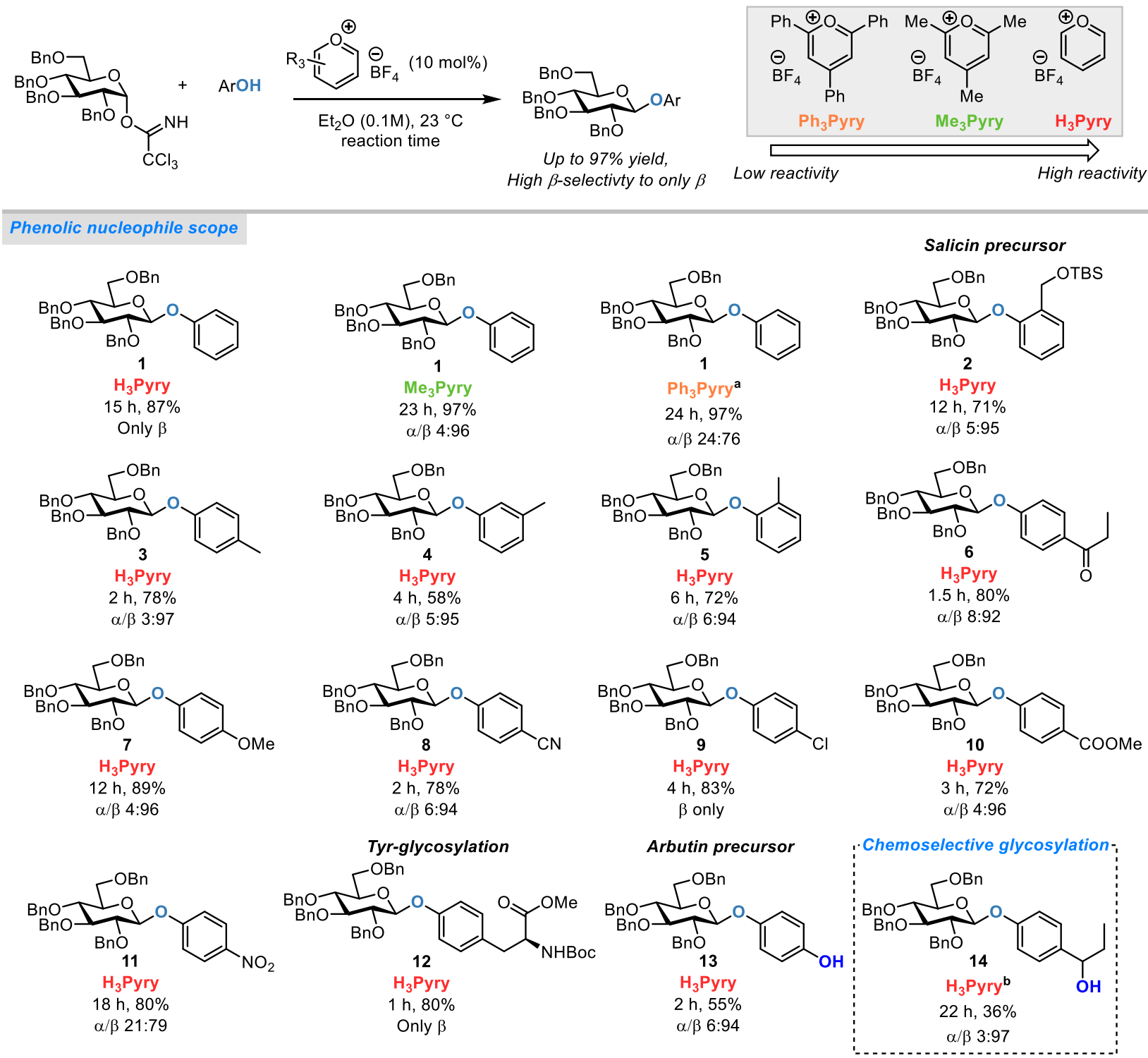

Scheme 1: Scope of phenolic glycosylation using 1.2 equiv. of the phenolic nucleophile. All reactions were carried out under an air atmosphere in a sealed vial. Reactions were monitored by TLC and stopped when the electrophile was consumed. $\alpha / \beta$ ratio was determined by crude ${ }^{1} \mathrm{H}-\mathrm{NMR}$. Yields were determined after purification by flashcolumn chromatography. a) Solvent was toluene. b) Solvent was $t$-butyl methyl ether.

Next, it was investigated how $\mathbf{H}_{3}$ Pyry would perform in glycosylations with glycosyl nucleophiles and an L-serine side chain (Scheme 2). The glycosylation of a primary glycosyl acceptor proceeded in excellent yields and high selectivity but required deviation from the standard procedure as toluene and $\mathrm{MeCN}$ gave higher $\beta$-selectivity than in $\mathrm{Et}_{2} \mathrm{O}$ for this substrate (product 15). A sterically hindered, secondary glycosyl nucleophile was found to be relatively incompatible with this glycosylation strategy, giving the (1-3)-linked disaccharide (16) in a lower yield as a mixture of anomers. We 
believe that the current methodology could be optimized further for this type of substrate (and secondary glycosyl acceptors in general), but this was outside the scope of the current investigation.
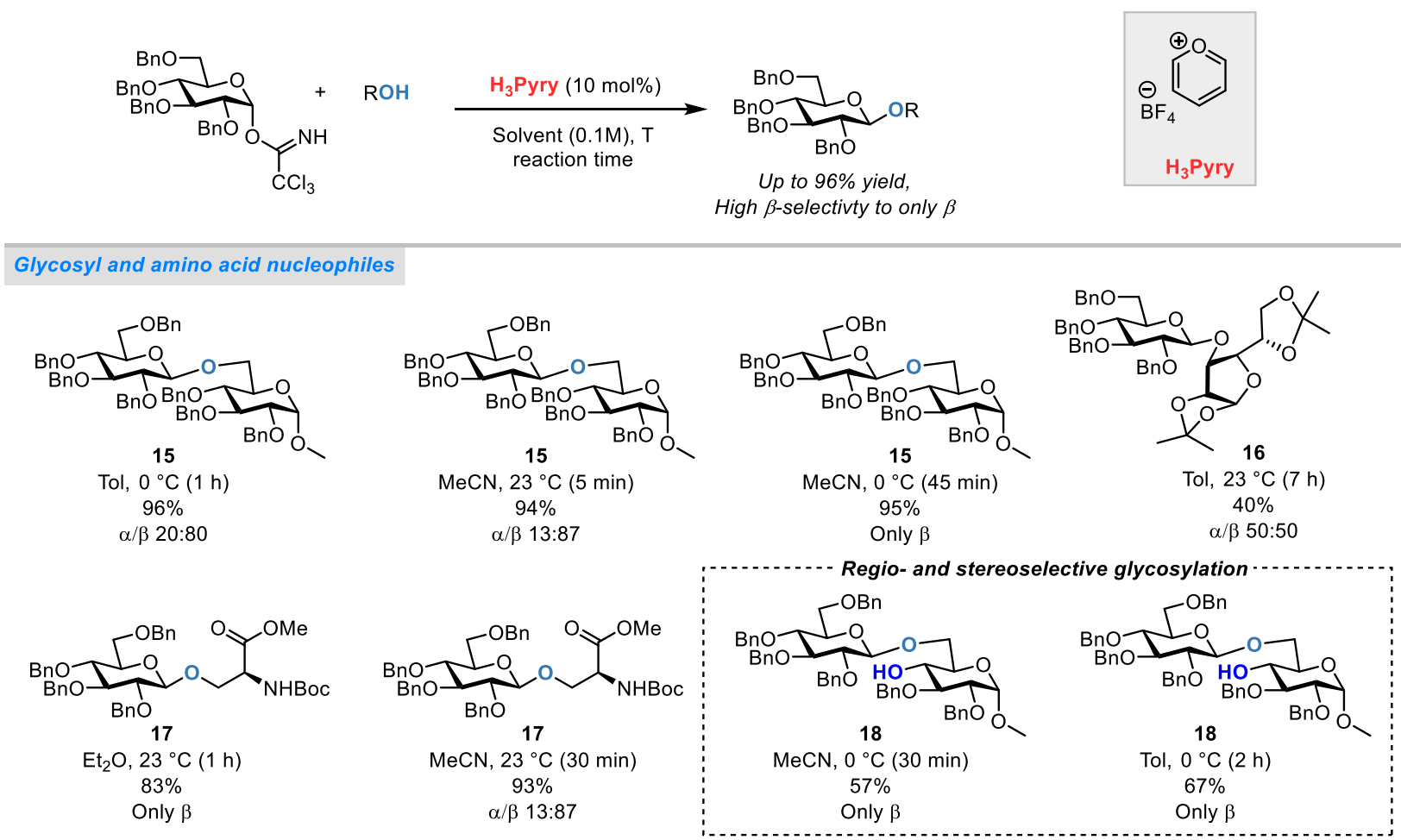

Scheme 2: Glycosylations of glycosyl nucleophiles and L-serine side chain using 1.2 equiv. of the nucleophile. All reactions were carried out under an air atmosphere in a sealed vial. $\alpha / \beta$ ratio was determined by crude ${ }^{1} \mathrm{H}-\mathrm{NMR}$. Yields were determined after purification by flash-column chromatography.

The glycosylation of an L-serine side chain (product 17) was found to proceed with similarly high degrees of $\beta$-selectivity and yield and furthermore gave high $\beta$-selectivity using the standard procedure in $\mathrm{Et}_{2} \mathrm{O}$ as well as when using $\mathrm{MeCN}$ as the solvent. Lastly, a 4,6-deprotected glycosyl nucleophile was glycosylated regioselectively with exclusive formation of the desired $\beta$-linked disaccharide (18) in both $\mathrm{MeCN}$ and in toluene.

Next, we investigated whether other parent sugars and other protective group schemes were tolerated during the glycosylation with $\mathbf{H}_{3}$ Pyry. We found that galactosylations (Scheme 3, products 19 and 20) proceeded with high $\beta$-stereoselectivity when the reactions were initiated at a lowered temperature and allowed to reach rt. Carrying out the galactosylationas at constant $\mathrm{T}$ will likely result in similar results, but an optimized procedure specifically for the galactosylations has not yet been completed. A 4,6-benzylidene-protected mannopyranosyl trichloroacetimidate (Scheme 3, middle) 
was also found to react with $\beta$-selectivity with a glycosyl nucleophile at $0{ }^{\circ} \mathrm{C}$ in both $\mathrm{Et}_{2} \mathrm{O}$ and toluene (product 21). This result was very promising as we expected that the steric congestion associated with the formation of 1,2-cis-glycosides to result significantly lower the selectivity.
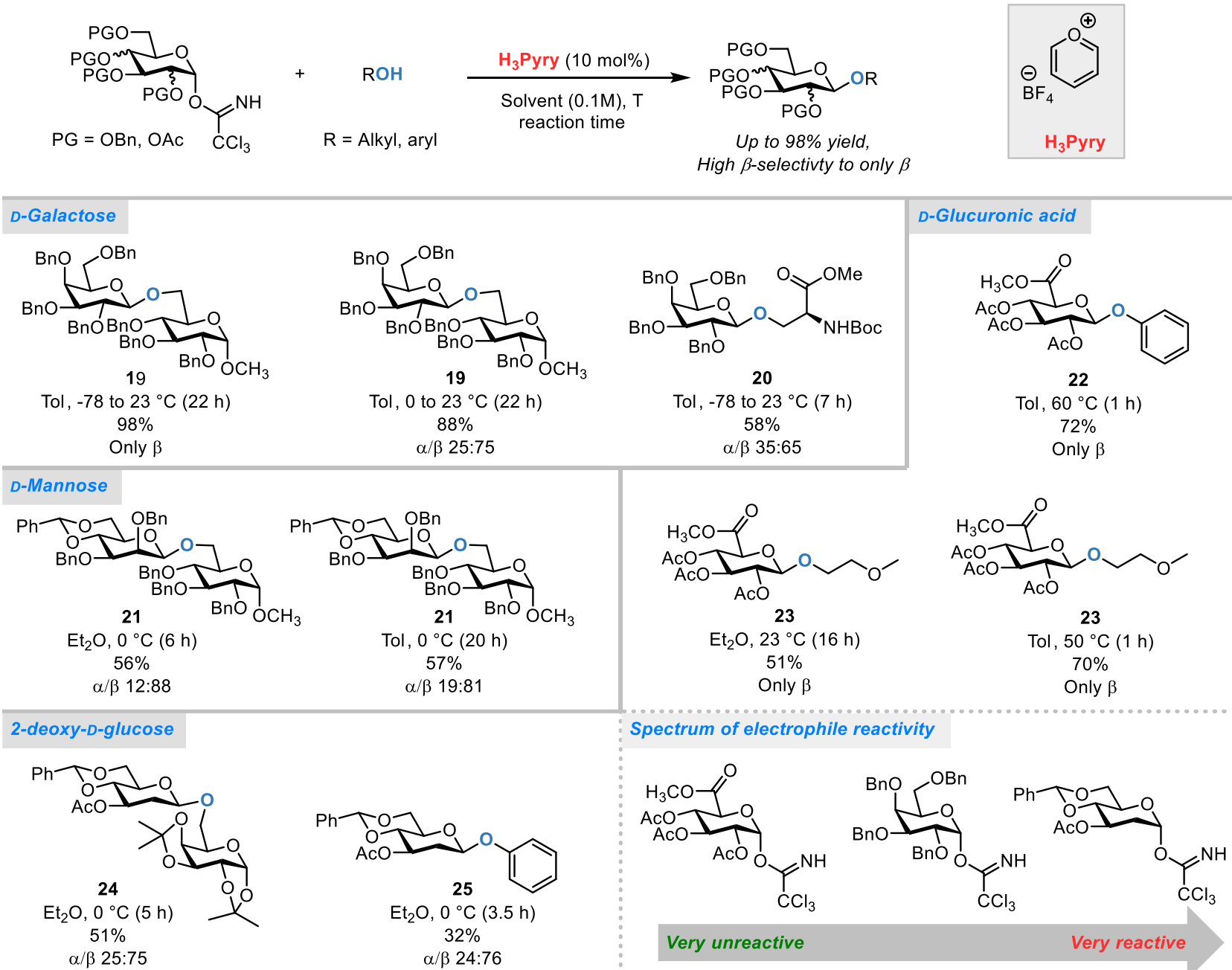

Scheme 3: Reaction scope using other parent sugar trichloroacetimidates as electrophiles with 1.2 equiv. of the nucleophile. All reactions were carried out under an air atmosphere in a sealed vial. $\alpha / \beta$ ratio was determined by crude ${ }^{1} \mathrm{H}-\mathrm{NMR}$. Yields were determined after purification by flash-column chromatography.

In an attempt to investigate how broad a reactivity spectrum of electrophiles were compatible with the current methodology, a very unreactive, acetyl-protected glucuronic acid trichloroacetimidate (products 22 and 23) and a highly reactive $\mathrm{e}^{48,49}$ 2-deoxy-glucopyranosyl trichloroacetimidate (products 24 and 25) were subjected to the glycosylation conditions (Scheme 3, top right and bottom). Although the electronically disarmed glucuronic acid electrophiles reacted at significantly lower rates than the other substrates, product formation did indeed proceed with exclusive formation of the desired $\beta$ - 
glycosides in good yields. Furthermore, heating the reactions to reduce reaction time was found to increase the yield whilst maintaining the high selectivity.

Stereoselective formation of 2-deoxy- $\beta$-glycosides remains one of the central challenges in the field of carbohydrate chemistry. ${ }^{8,50-54}$ To allow handling of 2-deoxy-glycosyl trichloroacetimidates, introduction of a disarming, conformationally restrictive 4,6-benzylidene tether was found to be a critical modification. We found that although the 4,6-benzylidene-protected 2-deoxy-glucosyl trichloroacetimidate was indeed compatible with the catalytic conditions, these reactions were less selective than with the fully oxygenated electrophiles (Scheme 3, products 22 and 23). Despite the lowered selectivity of the 2-deoxyglycosylation, we are very satisfied with how well this glycosylation methodology responds to significant changes to the reactivity of the substrates.

One of the major advantages of using trichloroacetimidates is the selective accessibility of either their $\alpha$ - or $\beta$-anomers, which has previously been utilized by Schmidt ${ }^{26-28,57-59}$ and ourselves. ${ }^{37}$ To investigate whether the pyrylium-catalysts also engaged in similar stereospecific glycosylations, we carried out a series of glucosylations and galactosylations (Scheme 4, top and middle) using the corresponding $\beta$-trichloroacetimidates. It was found that $\mathrm{Me}_{3}$ Pyry was in fact a superior catalyst over $\mathrm{H}_{3}$ Pyry for $\alpha$-glycosylations, resulting in high degrees of stereoselectivity for both parent sugars at ambient conditions.

Furthermore, we performed a competition experiment between different amino acids starting from a mixture of trichloroacetimidates (Scheme 4, bottom) to see if we could glycosylate one in the presence of another. We chose to use a mixture of the trichloroacetimidate for this experiment as we had previously established the stereospecific glycosylation of these substrates and wanted to make sure that both anomers would perform similarly. We anticipated that the primary alcohol of L-serine should out-compete both the secondary alcohol of L-threonine as well as the phenolic side chain of L-tyrosine as primary alcohols previously were glycosylated at very high reaction rates. In agreement with this hypothesis, we only isolated the serine glycosides from these experiments as anomeric mixtures that approximately mirrored that of the starting trichloroacetimidate. 

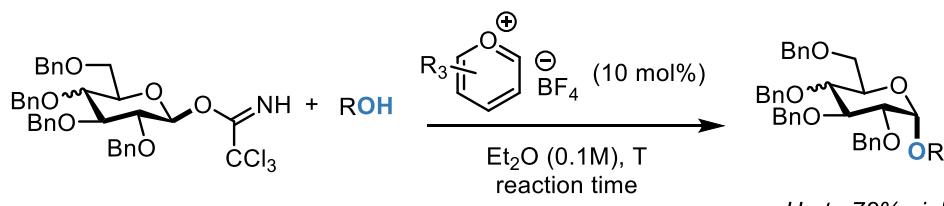

Up to $72 \%$ yield,

Moderate to high $\alpha$-selectivty

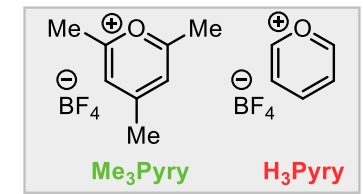

$\beta$-trichloroacetimidate
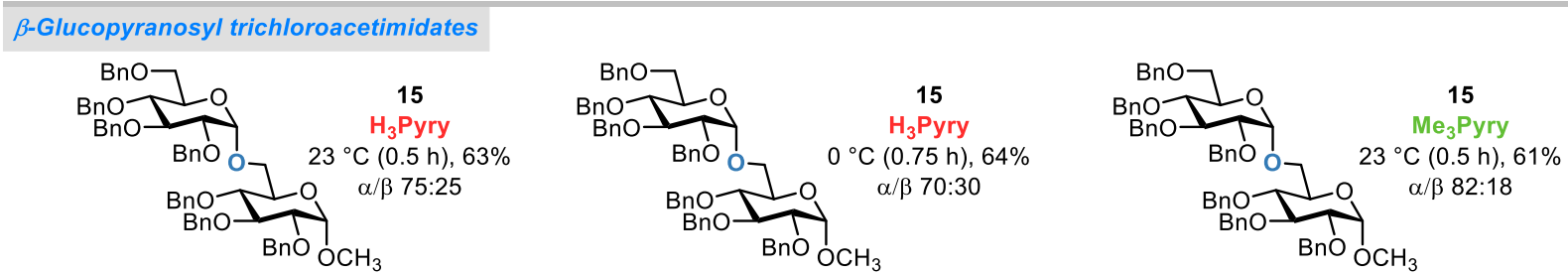

$\beta$-Galactoopyranosyl trichloroacetimidates
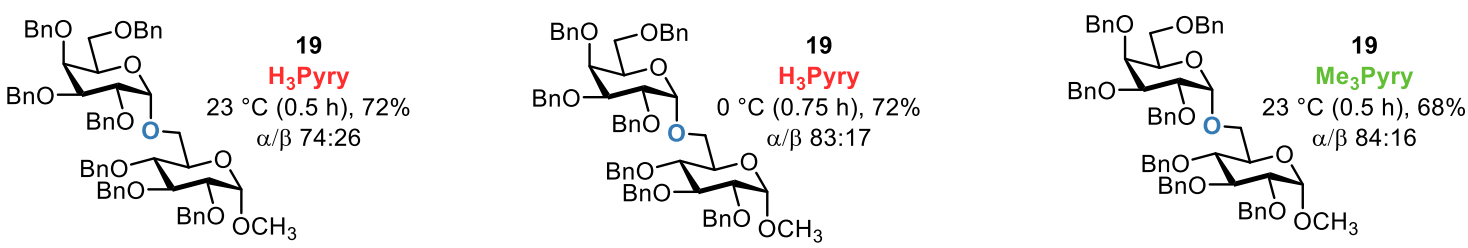

Competition experiments from TCA-mixtures

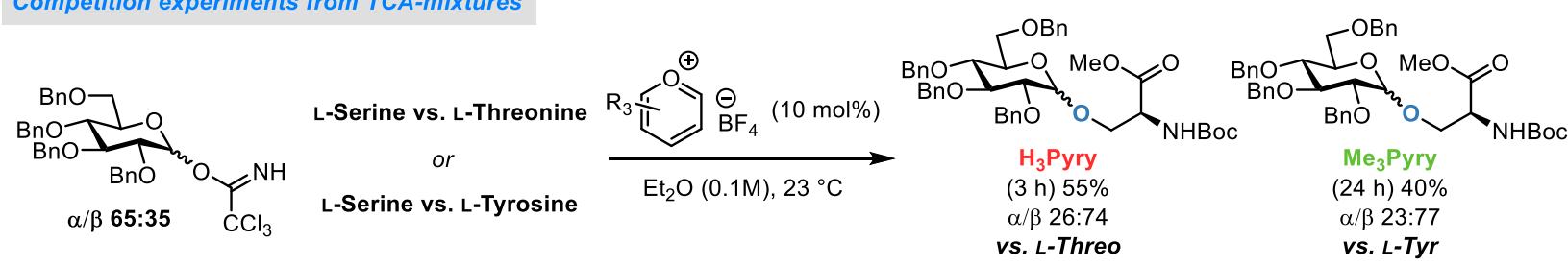

Scheme 4: Pyry-catalyzed glycosylations from $\beta$-trichloroacetimidates (top, middle) and competition experiment between pairs of amino acid side-chains from mixture of $\alpha / \beta$ trichloroacetimidates (bottom). Reactions were performed with 1.2 equiv. of the nucleophile. 1.2 equiv. of each nucleophile was used for the competition experiment. All reactions were carried out under an air atmosphere in a sealed vial. $\alpha / \beta$ ratio was determined by crude ${ }^{1} \mathrm{H}-\mathrm{NMR}$. All yields were determined after purification by flash-column chromatography.

\section{Mechanistic studies}

Our hypothesis for the pyrylium salt-catalyzed glycosylation was that the reactions proceeded through the formation of a Brønsted-acidic catalyst-nucleophile complex capable of activating the trichloroacetimidate. Based on our experience with the reaction, we expected the pyrylium salts to have very low affinity towards the trichloroacetimidate as the order of addition of the reactants was not found to influence the outcome of the glycosylations.

To study the mechanism of the pyrylium catalyzed glycosylation, a series of kinetic experiments were carried out using in-situ IR spectroscopy (ReactIR). For practical purposes, Me3Pyry was used for the mechanistic studies to avoid precipitation of the catalyst during the kinetic studies as this is 
significantly more soluble in organic solvents. We found that no consumption of the $\alpha$-glucosyl trichloroacetimidate ( $\alpha$-TCA) occurred in the presence of the Me3Pyry catalyst until a nucleophile was added (Figure S7). Furthermore, no rearrangement to the corresponding trichloroacectamide $N$ glycoside was observed. This supports our hypothesis of nucleophile activation and confirms the low affinity of Me3Pyry towards the trichloroacetimidate.

Next, the reaction order of each component was determined using 4-cyanophenol as the nucleophile as this did not overlap with other reactants in the IR spectrum. We observed first-order kinetics with respect to 4-cyanophenol (as evident from the linear fit in Figure 1, bottom left) and saturation kinetics for the $\boldsymbol{\alpha}$-TCA electrophile (Figure 1, bottom middle).

The glycosylations of phenolic nucleophiles presented above (Scheme 4) revealed that phenols with electron-donating and-withdrawing substituents gave rise to quite similar yields and selectivity. In order to investigate this behavior, we established a linear free-energy relationship using a series of phenolic nucleophiles with different substituents. From the Hammett plot, (Figure 1, bottom right) it was evident that both electron-donating and -withdrawing substituents were rate accelerating, while the mildly electron-donating methyl substituent gave rise to the lowest reaction rate amongst the nucleophiles included in the plot. The non-linear free energy relationship indicates that the identity of the rate-determining step (RDS) is dependent on the electronic properties of the phenolic nucleophile. 

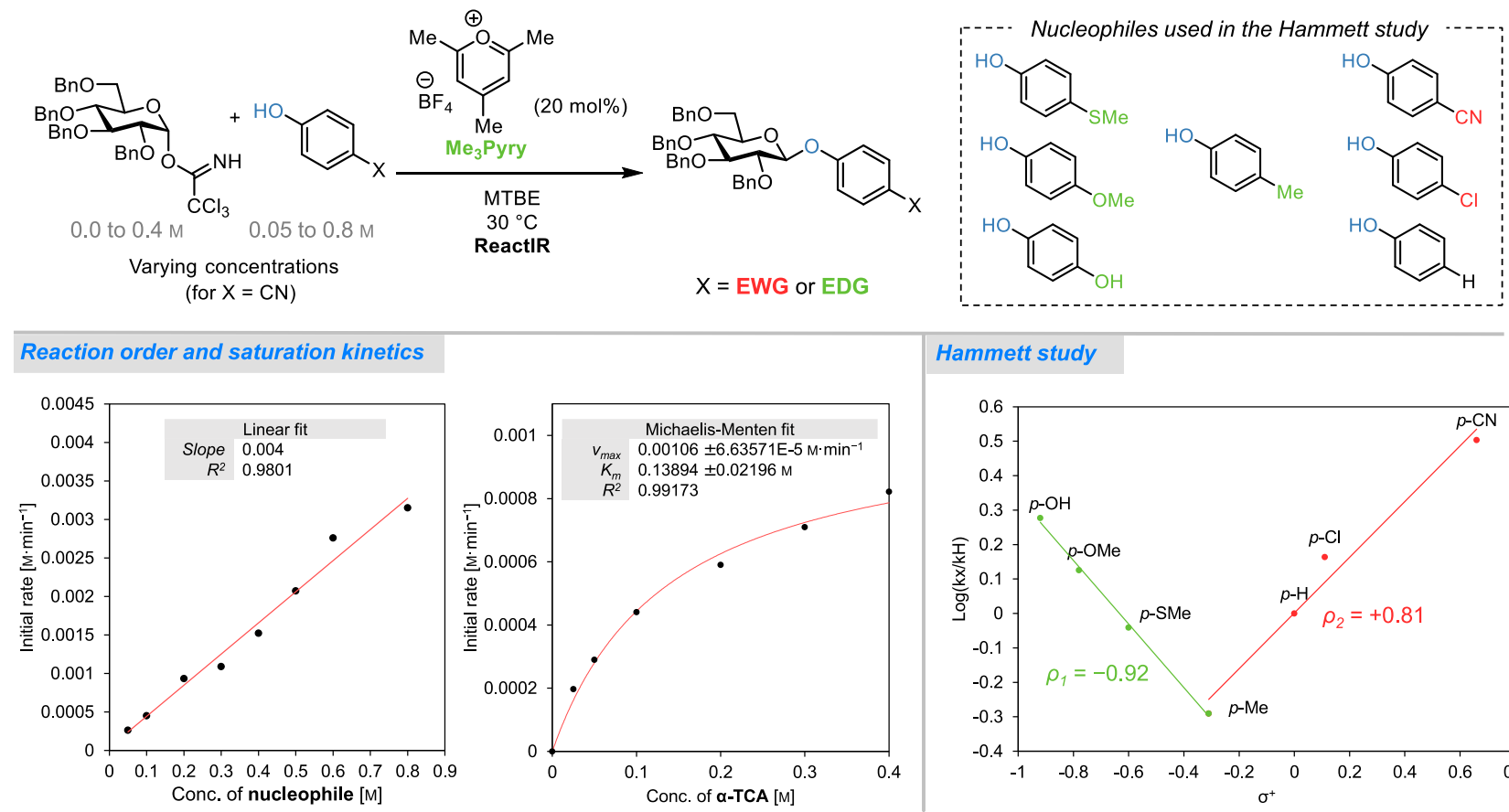

Hammett study

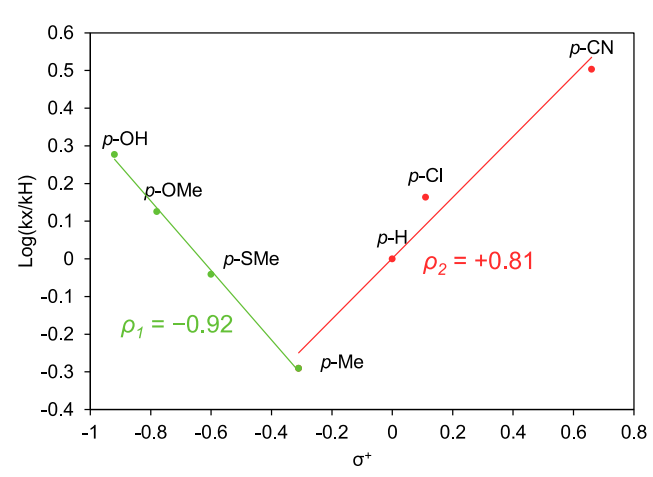

Figure 2: Mechanistic study of the $\mathrm{Me}_{3}$ Pyry catalyzed phenolic $O$-glucosylation (top left) based on kinetic experiments. For determination of reaction order, the concentration of the catalyst and either the nucleophile (4cyanophenol) or the electrophile $\alpha$-TCA was maintained at $0.1 \mathrm{M}$ while varying the concentration of either the nucleophile (bottom, left) or the electrophile (bottom, middle). For the Hammett plot in the bottom left corner seven different phenolic nucleophiles were used (top, right).

Next, control experiments to probe the interactions between the pyrylium catalyst and the nucleophile were undertaken. Berkessel and co-workers recently reported a pyridinium-catalyzed activation of glycals for a highly $\alpha$-selective 2-deoxyglycosylation, which was found to take place via in situ-formation of a hemiaminal intermediate between the catalyst and the nucleophile. ${ }^{60}$ However, H3Pyry was unable to activate an armed glucal when using phenol as the nucleophile, indicating that the $\mathbf{H}_{3} \mathbf{P} \mathbf{P r y}$-nucleophile complex was not sufficiently acidic to protonate the glucal double bond (Figure 3). Furthermore, it was investigated whether an acetal intermediate could be isolated by treating a mixture of nucleophile and catalyst with base as was previously accomplished by the Berkessel team when investigating their pyridinium ion-catalyzed glycosylations. ${ }^{60}$ However, we did not observe any formation of an acetal intermediate (Figure 3, middle), indicating the nucleophile-catalyst interaction has significantly less covalent character than what was observed with the pyridinium ion catalysts. We furthermore conducted an experiment using 1.0 and 5.0 equiv. of the catalyst to investigate whether a large excess of catalyst would deplete the nucleophile from 
solution due to acetal-formation and thus lower the reaction rate. However, we found that increasing the concentration of the catalyst led to a higher reaction rate, which rules out the possibility that small amounts of a non-nucleophilic catalyst-nucleophile acetal intermediate was acting as the actual catalyst. We can rule out that the active catalytic species is not a complex of the trichloroacetamide leaving group and the pyrylium salts, yielding pyridinium intermediates analogous the those reported by Cornella and co-workers (vide supra), as we do not observe an induction period for the reaction during the kinetic studies. In addition, we have not observed the autocatalytic kinetic profile that should be associated with the active catalytic species containing the leaving group as reported during glycosylations of glycosyl fluorides. ${ }^{61}$

We believe that our mechanistic observations (1) that the reaction is stereospecific, (2) that the reaction is ordered in both electrophile and nucleophile and, (3) that we see a nonlinear concave upwards Hammett plot, is best reconciled with an $S_{\mathrm{N}} 2$-like substitution of the protonated trichloroacetimidate electrophile (Figure 3, bottom), i.e. an $S_{\mathrm{N}} 2$ reaction that requires a protonation step followed by a nucleophilic substitution of the leaving group. 


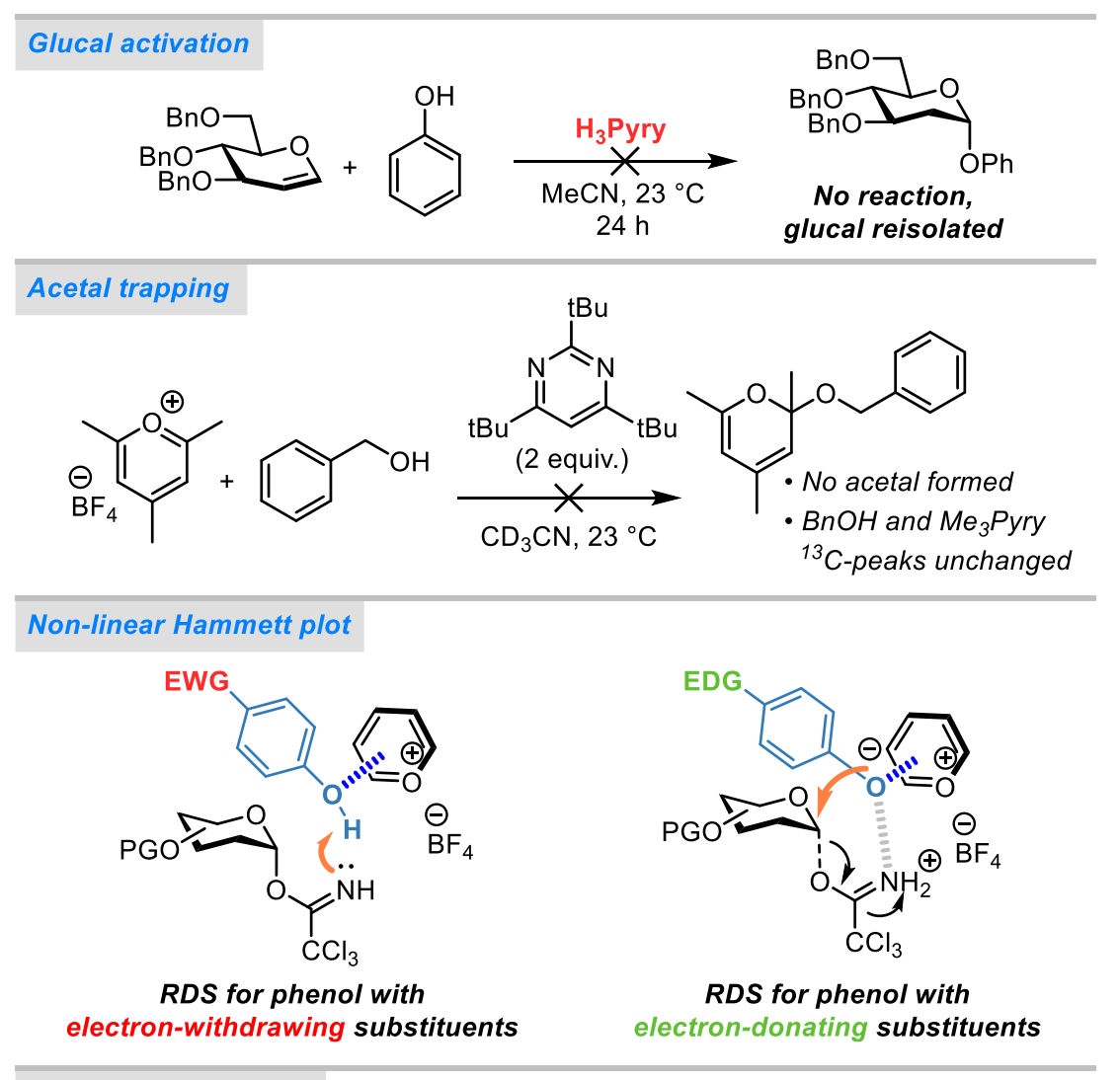

Proposed mechanism

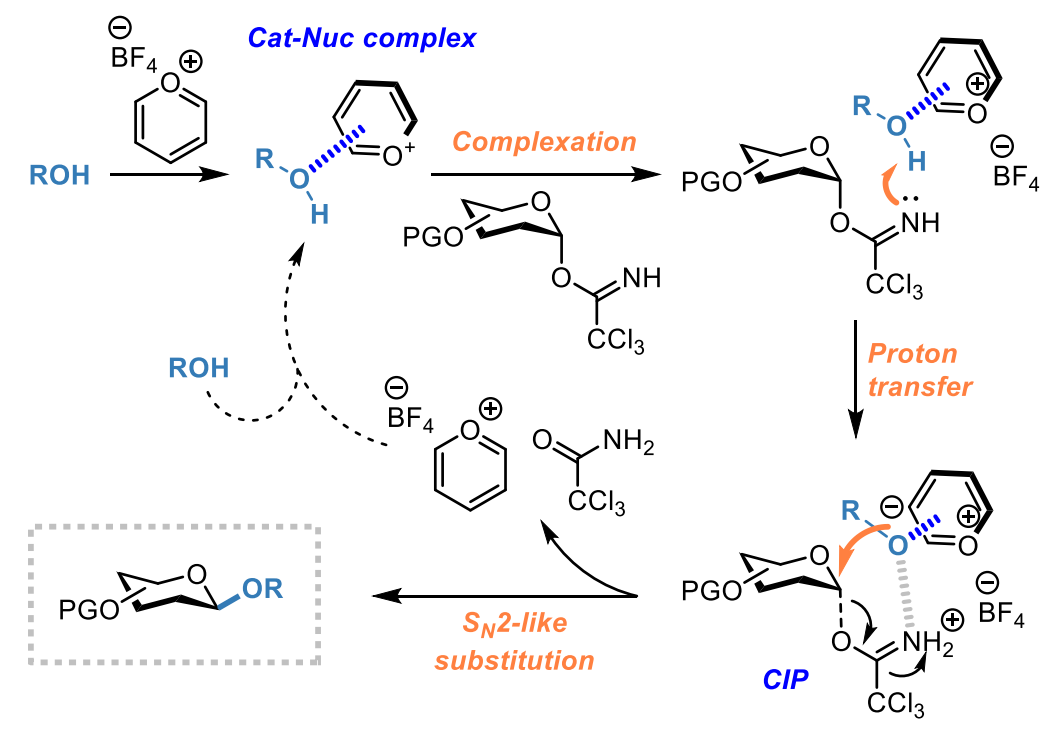

Figure 3: Top: Attempted glucal activation as previously shown possible with pyrylium ion catalysts. ${ }^{60}$ Middle: Acetal trapping experiment to probe catalyst-nucleophile as inspired by Berkessel and co-workers. ${ }^{60}$ Bottom: Proposed mechanism for the pyrylium-catalyzed glycosylation of trichloroacetimidates. CIP = Contact ion pair. 


\section{Conclusions}

In conclusion, we have developed a highly selective glycosylation method which allows glycosylations of a wide range of phenolic nucleophiles as well as several glycosyl- and amino acid nucleophiles. The reaction setup is extraordinarily simple as it takes place at ambient or near-ambient conditions under air and requires no specialized equipment, which should render this chemistry approachable by carbohydrate chemists and non-specialists alike. The fact that no reaction takes place prior to adding mixing all three reaction components should render this particular glycosylation strategy much less dependent on the skills and experience of the operator, making this chemistry accessible by scientists of various backgrounds.

We have performed the glycosylations with electrophiles from several different parent sugars that represent a wide spectrum of reactivity, which underlines the potential of this methodology. Furthermore, the $\alpha$ - and $\beta$-trichloroacetimidate electrophiles are readily available via robust, highyielding literature procedures and they are shelf-stable for at least several weeks when stored in a fridge. Mechanistically, we believe that the glycosylation occurs via two steps in tandem. Initially, the nucleophile-catalyst complex protonates the trichloroacetimidate, leading to a contact-ion-pair. The following $S_{\mathrm{N}} 2$-substitution of the protonated leaving group yields the $O$-glycoside with inversion of the anomeric configuration. The H3Pyry and Me3Pyry catalysts offer incredibly easy access to a wide range of $\beta$-linked phenyl glycosides as well as relevant alkyl glycosides, which could be of great value to the glycoscience community.

\section{References}

(1) Walt, D. R.; Aoki-Kinoshita, K. F.; Bendiak, B.; Bertozzi, C. R. .; Boons, G.-J.; Darvill, A.; Hart, G. W.; Kiessling, L. L.; Lowe, J. . M.; R.; Paulson, J.; Sasisekharan, R.; Varki, A. P.; Wong, C.-H. Transforming Glycoscience: A Roadmap for the Future; The National Academies Press: Washington, DC, 2012.

(2) Zhu, X.; Schmidt, R. R. New Principles for Glycoside-Bond Formation. Angew. Chem., Int. Ed. 2009, 48, 1900-1934.

(3) Demchenko, A. V. Stereoselective Chemical 1,2-Cis O-Glycosylation: From 'Sugar Ray' to Modern Techniques of the 21st Century. Synlett 2003, 2003, 1225-1240.

(4) Nielsen, M. M.; Pedersen, C. M. Catalytic Glycosylations in Oligosaccharide Synthesis. Chem. 
Rev. 2018, 118, 8285-8358.

(5) Kulkarni, S. S.; Wang, C.-C.; Sabbavarapu, N. M.; Podilapu, A. R.; Liao, P.-H.; Hung, S.-C. "One-Pot" Protection, Glycosylation, and Protection-Glycosylation Strategies of Carbohydrates. Chem. Rev. 2018, 118, 8025-8104.

(6) Seeberger, P. H. The Logic of Automated Glycan Assembly. Acc. Chem. Res. 2015, 48, 14501463.

(7) Panza, M.; Pistorio, S. G.; Stine, K. J.; Demchenko, A. V. Automated Chemical Oligosaccharide Synthesis: Novel Approach to Traditional Challenges. Chem. Rev. 2018, 118, 8105-8150.

(8) Bennett, C. S.; Galan, M. C. Methods for 2-Deoxyglycoside Synthesis. Chem. Rev. 2018, 118, 7931-7985.

(9) Schmidt, R. R.; Michel, J. Facile Synthesis of $\alpha$ - and $\beta$-O-Glycosyl Imidates; Preparation of Glycosides and Disaccharides. Angew. Chem., Int. Ed. 1980, 19, 731-732.

(10) Bohé, L.; Crich, D. A Propos of Glycosyl Cations and the Mechanism of Chemical Glycosylation; the Current State of the Art. Carbohydr. Res. 2015, 403, 48-59.

(11) Adero, P. O.; Amarasekara, H.; Wen, P.; Bohé, L.; Crich, D. The Experimental Evidence in Support of Glycosylation Mechanisms at the SN1-SN2 Interface. Chem. Rev. 2018, 118, 8242-8284.

(12) Crich, D.; Sun, S. Formation of $\beta$-Mannopyranosides of Primary Alcohols Using the Sulfoxide Method. J. Org. Chem. 1996, 61, 4506-4507.

(13) Crich, D.; Sun, S. Are Glycosyl Triflates Intermediates in the Sulfoxide Glycosylation Method? A Chemical and 1H, 13C, and 19F NMR Spectroscopic Investigation. J. Am. Chem. Soc. 1997, 119, 11217-11223.

(14) Crich, D. Mechanism of a Chemical Glycosylation Reaction. Acc. Chem. Res. 2010, 43, 11441153.

(15) Jia, X. G.; Demchenko, A. V. Intramolecular Glycosylation. Beilstein J. Org. Chem. 2017, 13, 2028-2048.

(16) Ishiwata, A.; Lee, Y. J.; Ito, Y. Recent Advances in Stereoselective Glycosylation through Intramolecular Aglycon Delivery. Org. Biomol. Chem. 2010, 8, 3596-3608.

(17) Nakagawa, A.; Tanaka, M.; Hanamura, S.; Takahashi, D.; Toshima, K. Regioselective and 1,2Cis- $\alpha$-Stereoselective Glycosylation Utilizing Glycosyl-Acceptor-Derived Boronic Ester Catalyst. Angew. Chem., Int. Ed. 2015, 54, 10935-10939. 
(18) Tanaka, M.; Takahashi, D.; Toshima, K. 1,2-Cis- $\alpha$-Stereoselective Glycosylation Utilizing a Glycosyl-Acceptor-Derived Borinic Ester and Its Application to the Total Synthesis of Natural Glycosphingolipids. Org. Lett. 2016, 18, 5030-5033.

(19) Tanaka, M.; Nashida, J.; Takahashi, D.; Toshima, K. Glycosyl-Acceptor-Derived Borinic Ester-Promoted Direct and $\beta$-Stereoselective Mannosylation with a 1,2-Anhydromannose Donor. Org. Lett. 2016, 18, 2288-2291.

(20) Nishi, N.; Nashida, J.; Kaji, E.; Takahashi, D.; Toshima, K. Regio- and Stereoselective $\beta$ Mannosylation Using a Boronic Acid Catalyst and Its Application in the Synthesis of a Tetrasaccharide Repeating Unit of Lipopolysaccharide Derived from E. Coli O75. Chem. Commun. 2017, 53, 3018-3021.

(21) Nishi, N.; Sueoka, K.; Iijima, K.; Sawa, R.; Takahashi, D.; Toshima, K. Stereospecific $\beta-1-$ Rhamnopyranosylation through an SNi-Type Mechanism by Using Organoboron Reagents. Angew. Chem., Int. Ed. 2018, 57, 13858-13862.

(22) Tanaka, M.; Nakagawa, A.; Nishi, N.; Iijima, K.; Sawa, R.; Takahashi, D.; Toshima, K. Boronic-Acid-Catalyzed Regioselective and 1,2-Cis-Stereoselective Glycosylation of Unprotected Sugar Acceptors via SNi-Type Mechanism. J. Am. Chem. Soc. 2018, 140, 36443651.

(23) Schmidt, R. R.; Gaden, H.; Jatzke, H. New Catalysts for the Glycosyl Transfer with OGlycosyl Trichloroacetimidates. Tetrahedron Lett. 1990, 31, 327-329.

(24) Kumar, A.; Schmidt, R. R. Reversal of Anomeric Selectivity with O-Glycosyl Trichloroacetimidates as Glycosyl Donors and Thiols as Acceptors Under Acid/Base Catalysis. Eur. J. Org. Chem. 2012, 2012, 2715-2719.

(25) Geng, Y.; Kumar, A.; Faidallah, H. M.; Albar, H. A.; Mhkalid, I. A.; Schmidt, R. R. Cooperative Catalysis in Glycosidation Reactions with O-Glycosyl Trichloroacetimidates as Glycosyl Donors. Angew. Chem., Int. Ed. 2013, 52, 10089-10092.

(26) Kumar, A.; Kumar, V.; Dere, R. T.; Schmidt, R. R. Glycoside Bond Formation via Acid-Base Catalysis. Org. Lett. 2011, 13, 3612-3615.

(27) Peng, P.; Schmidt, R. R. An Alternative Reaction Course in O-Glycosidation with O-Glycosyl Trichloroacetimidates as Glycosyl Donors and Lewis Acidic Metal Salts as Catalyst: AcidBase Catalysis with Gold Chloride-Glycosyl Acceptor Adducts. J. Am. Chem. Soc. 2015, 137, 12653-12659.

(28) Kumar, A.; Geng, Y.; Schmidt, R. R. Silicon Fluorides for Acid-Base Catalysis in 
Glycosidations. Adv. Synth. Catal. 2012, 354, 1489-1499.

(29) Gouliaras, C.; Lee, D.; Chan, L.; Taylor, M. S. Regioselective Activation of Glycosyl Acceptors by a Diarylborinic Acid-Derived Catalyst. J. Am. Chem. Soc. 2011, 133, 1392613929.

(30) Beale, T. M.; Moon, P. J.; Taylor, M. S. Organoboron-Catalyzed Regio- and Stereoselective Formation of $\beta$-2-Deoxyglycosidic Linkages. Org. Lett. 2014, 16, 3604-3607.

(31) D’Angelo, K. A.; Taylor, M. S. Borinic Acid-Catalyzed Stereo- and Site-Selective Synthesis of $\beta$-Glycosylceramides. Chem. Commun. 2017, 53, 5978-5980.

(32) Kimura, T.; Sekine, M.; Takahashi, D.; Toshima, K. Chiral Brønsted Acid Mediated Glycosylation with Recognition of Alcohol Chirality. Angew. Chem., Int. Ed. 2013, 52, 1213112134.

(33) Park, Y.; Harper, K. C.; Kuhl, N.; Kwan, E. E.; Liu, R. Y.; Jacobsen, E. N. Macrocyclic BisThioureas Catalyze Stereospecific Glycosylation Reactions. Science 2017, 355, 162-166.

(34) Levi, S. M.; Li, Q.; Rötheli, A. R.; Jacobsen, E. N. Catalytic Activation of Glycosyl Phosphates for Stereoselective Coupling Reactions. Proc. Natl. Acad. Sci. 2019, 116, 35-39.

(35) Mayfield, A. B.; Metternich, J. B.; Trotta, A. H.; Jacobsen, E. N. Stereospecific Furanosylations Catalyzed by Bis-Thiourea Hydrogen-Bond Donors. J. Am. Chem. Soc. 2020, 142, 4061-4069.

(36) Li, Q.; Levi, S. M.; Jacobsen, E. N. Highly Selective $\beta$-Mannosylations and $\beta$ Rhamnosylations Catalyzed by Bis-Thiourea. J. Am. Chem. Soc. 2020, 142, 11865-11872.

(37) Nielsen, M. M.; Mała, P.; Baldursson, E. P.; Pedersen, C. M. Self-Promoted and Stereospecific Formation of N-Glycosides. Chem. Sci. 2019, 10, 5299-5307.

(38) Pinna, A.; Pedersen, C. M. Chemoselectivity in Self-Promoted Glycosylation: N- vs. OGlycosylation. Eur. J. Org. Chem. 2020, 2020, 3914-3917.

(39) Beltrán, A.; Burguete, M. I.; Galindo, F.; Luis, S. V. Synthesis of New Fluorescent Pyrylium Dyes and Study of Their Interaction with N-Protected Amino Acids. New J. Chem. 2020, 44, 9509-9521.

(40) Maeda, T.; Nitta, S.; Nakao, H.; Yagi, S.; Nakazumi, H. Squaraine Dyes with Pyrylium and Thiopyrylium Components for Harvest of Near Infrared Light in Dye-Sensitized Solar Cells. J. Phys. Chem. C 2014, 118, 16618-16625.

(41) Nicewicz, D. A.; Nguyen, T. M. Recent Applications of Organic Dyes as Photoredox Catalysts in Organic Synthesis. ACS Catal. 2014, 4, 355-360. 
(42) Miranda, M. A.; Garcia, H. 2,4,6-Triphenylpyrylium Tetrafluoroborate as an Electron-Transfer Photosensitizer. Chem. Rev. 1994, 94, 1063-1089.

(43) Romero, N. A.; Nicewicz, D. A. Organic Photoredox Catalysis. Chem. Rev. 2016, 116, 1007510166.

(44) Moser, D.; Duan, Y.; Wang, F.; Ma, Y.; O’Neill, M. J.; Cornella, J. Selective Functionalization of Aminoheterocycles by a Pyrylium Salt. Angew. Chem., Int. Ed. 2018, 57, 11035-11039.

(45) Pérez-Palau, M.; Cornella, J. Synthesis of Sulfonyl Fluorides from Sulfonamides. Eur. J. Org. Chem. 2020, 2020, 2497-2500.

(46) Ma, Y.; Pang, Y.; Chabbra, S.; Reijerse, E. J.; Schnegg, A.; Niski, J.; Leutzsch, M.; Cornella, J. Radical C-N Borylation of Aromatic Amines Enabled by a Pyrylium Reagent. Chem. - A Eur. J. 2020, 26, 3738-3743.

(47) Schäffer, C.; Dietrich, K.; Unger, B.; Scheberl, A.; Rainey, F. A.; Kählig, H.; Messner, P. A Novel Type of Carbohydrate-Protein Linkage Region in the Tyrosine-Bound S-Layer Glycan of Thermoanaerobacterium Thermosaccharolyticum D120-70. Eur. J. Biochem. 2000, 267, 5482-5492.

(48) Chen, J.-H.; Ruei, J.-H.; Mong, K.-K. T. Iterative $\alpha$-Glycosylation Strategy for 2-Deoxy- and 2,6-Dideoxysugars: Application to the One-Pot Synthesis of Deoxysugar-Containing Oligosaccharides. Eur. J. Org. Chem. 2014, 2014, 1827-1831.

(49) Zhang, Z.; Ollmann, I. R.; Ye, X.-S.; Wischnat, R.; Baasov, T.; Wong, C.-H. Programmable One-Pot Oligosaccharide Synthesis. J. Am. Chem. Soc. 1999, 121, 734-753.

(50) Mizia, J. C.; Bennett, C. S. Reagent Controlled Direct Dehydrative Glycosylation with 2Deoxy Sugars: Construction of the Saquayamycin Z Pentasaccharide. Org. Lett. 2019, 21, 5922-5927.

(51) Issa, J. P.; Bennett, C. S. A Reagent-Controlled SN2-Glycosylation for the Direct Synthesis of $\beta$-Linked 2-Deoxy-Sugars. J. Am. Chem. Soc. 2014, 136, 5740-5744.

(52) Lloyd, D.; Bennett, C. S. An Improved Approach to the Direct Construction of 2-Deoxy- $\beta$ Linked Sugars: Applications to Oligosaccharide Synthesis. Chem. - A Eur. J. 2018, 24, 76107614.

(53) Hoang, K. M.; Lees, N. R.; Herzon, S. B. Programmable Synthesis of 2-Deoxyglycosides. J. Am. Chem. Soc. 2019, 141, 8098-8103.

(54) Hoang, K. M.; Lees, N. R.; Herzon, S. B. General Method for the Synthesis of $\alpha$ - or $\beta$ Deoxyaminoglycosides Bearing Basic Nitrogen. J. Am. Chem. Soc. 2021, 143, 2777-2783. 
(55) Mootoo, D. R.; Konradsson, P.; Udodong, U.; Fraser-Reid, B. Armed and Disarmed NPentenyl Glycosides in Saccharide Couplings Leading to Oligosaccharides. J. Am. Chem. Soc. 1988, 110, 5583-5584.

(56) Fraser-Reid, B.; Wu, Z.; Udodong, U. E.; Ottosson, H. Armed/Disarmed Effects in Glycosyl Donors: Rationalization and Sidetracking. J. Org. Chem. 1990, 55, 6068-6070.

(57) Kumar, A.; Schmidt, R. R. Reversal of Anomeric Selectivity with O-Glycosyl Trichloroacetimidates as Glycosyl Donors and Thiols as Acceptors Under Acid/Base Catalysis. Eur. J. Org. Chem. 2012, 2715-2719.

(58) Schmidt, R. R.; Stumpp, M. Glycosylimidate, 10. Glycosylphosphate Aus Glycosyl(Trichloracetimidaten). Liebigs Ann. 1984, 1984, 680-691.

(59) Schmidt, R. R.; Stumpp, M.; Michel, J. $\alpha$ - and $\beta$-D-Glucopyranosyl Phosphates from $\alpha$-DGlucoypyranosyl Trichloroacetimidates. Tetrahedron Lett. 1982, 23, 405-408.

(60) Das, S.; Pekel, D.; Neudörfl, J.-M.; Berkessel, A. Organocatalytic Glycosylation by Using Electron-Deficient Pyridinium Salts. Angew. Chem., Int. Ed. 2015, 54, 12479-12483.

(61) Nielsen, M. M.; Qiao, Y.; Wang, X.; Pedersen, C. M., Vessel Effect in C-F Bond Activation Prompts Revised Mechanism and Reveals an Autocatalytic Glycosylation. Eur. J. Org. Chem. 2020, 2020, 140-144 